\title{
ASPECTS BIOLOGIQUES DANS L'ETUDE DU BASSIN DE L'YSER
}

\author{
Par G. VANHOOREN, \\ Institut d'Hygiène et d'Epidémiologie \\ du Département de l'Environnement à Bruxelles
}

\section{RESUME}

L'examen hydrobiologique de l'Yser est axé principalement sur deux aspects : d'une part, celui de l'état trophique et d'autre part celui de la pollution de l'eau courante.

En général, on se trouve en présence d'un écosystème en état de pollution organique, aussi bien exogène qu'autogène, cette pollution autogène étant provoquée par une eutrophisation fort avancée. Cet ètat, accentué par les caractéristiques hydrodynamiques du fleuve, s'explique par l'abondance des éléments nutritifs (composés azotés et phosphates).

Les phénomènes biologiques qui en découlent donnent lieu à des situa. tions d'anaérobiose en certains endroits et expliquent la détérioration du Bassin de I'Yser.

\section{INTRODUCTION}

Précédemment, I'Yser ètait connu comme une rivière saine et fort poissonneuse. Au cours des dernières années, on y a signalé périodiquement des mortalités de poissons.

Récemment, l'Yser et ses affluents ont été étudiès par le groupe *Inventaire de la Pollution des eaux", créé sous l'égide de la Commission interministérielle de la Programmation scientifique, au sein du projet national de R.D. sur l'environnement physique et biologique - Eau. Ce groupe est constitué d'équipes de l'Institut d'Hygiène et d'Epidémiologie (Ministère de la Santé publique et de la Famille) et de l'Institut de Recherches chimiques (Ministère de l'Agriculture)

Les résultats ont èté publiés fin 1973 (1). 


\section{CARACTERISTIQUES DU BASSIN DE L'YSER}

L'Yser est un fleuve de faible importance. Venant de France, où il a parcouru $37 \mathrm{~km}$ sur les 78 de son cours, il traverse lentement une région essentiellement agricole, de faible densitè de population (180 hab./ $\mathrm{km}^{2}$ ) et peu industrialisée. En Belgique, il reçoit plusieurs affluents et il est séparé du chenal da Nieuport par une écluse (Fig. 1).

Le courant de l'Yser est toujours faible, la dénivellation du bassin ne dépassant pas 5 mètres. A proximité de l'embouchure, les mouvements des masses d'eau se font aussi bien dans un sens que dans l'autre au gré des éclusages associés aux marées. De plus, certains facteurs influencent la vitesse d'écoulement.

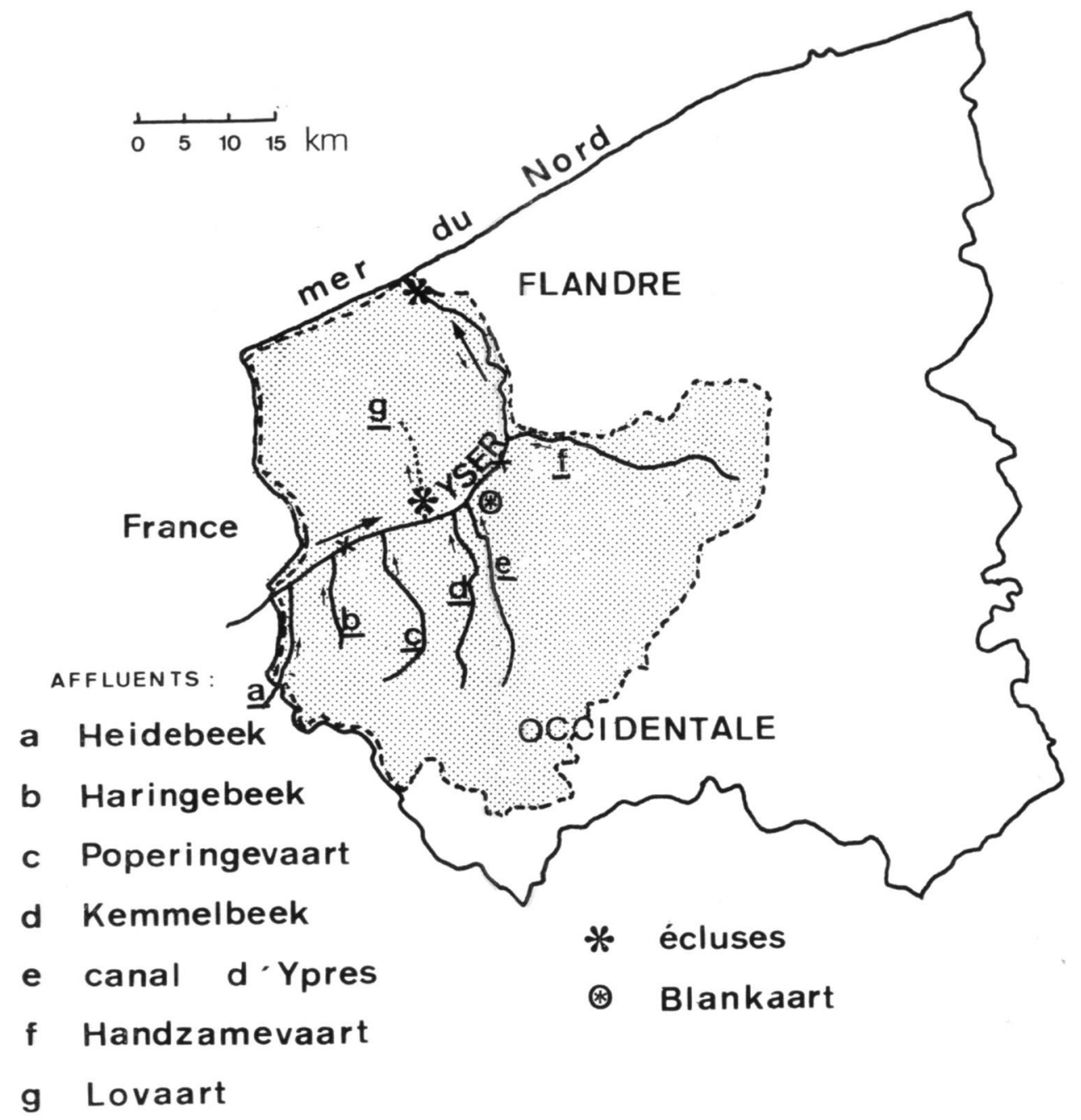

Fig. 1 - Bassin de l'Yser : présentation géographique. 
En période de hautes eaux, le courant de l'Yser est ralenti par l'ouverture du Lo-vaart, bifurcation servant de trop-plein à I'Yser supérieur, et par pompage des eaux aux alentours de l'étang du Blankaart pour l'alimentation de ce bassin d'épargne.

Ces différentes raisons expliquent qu'on ne peut déterminer dans quelle mesure l'Yser débite en mer.

L'Yser reçoit peu de décharges; celles-ci sont plus nombreuses en bordure des affluents.

Les débits des affluents par rapport à celui de I'Yser ont été estimés au 1/4 pour l'Heidebeek, au 1/25 pour l'Haringebeek, au 1/12 pour le canal de Poperinge, au $1 / 3$ pour le canal d'Ypres, au $1 / 6$ pour l'Handzamevaart.

\section{PRESENTATION DES RESULTATS}

Les analyses physico-chimiques de l'eau et des sédiments à différentes périodes de l'année n'ont pas mis en évidence de pollution par des produits toxiques, mais une accumulation de substances nutritives a été observée dans tous les échantillons: composés azotés et surtout phosphates. Les teneurs en azote total atteignent, à certains endroits, près de $25 \mathrm{mg} / \mathrm{l}$, celles en phosphore plus de $10 \mathrm{mg} \mathrm{PO} 4^{3-} / /$ dans I'Yser et beaucoup plus encore dans les affluents.

L'examen hydrobiologique, l'étude du plancton et l'étude de la structure des biocénoses se développant sur des substrats artificiels standardisés (3) ont permis, au moyen d'indicateurs biologiques, d'évaluer la qualité moyenne de l'eau et d'obtenir des informations concernant la pollution de I'Yser. Nous avons utilisé le système des valences saprobiques (ZELINKA \& MARVAN, SLADECEK (4) (5), permettant de se former une idée de la situation sur une certaine période.

La situation momentannée a été évaluée par le système de l'index étendu selon SLADECEK (6), basé sur les données physico-chimiques et bactériologiques.

Les observations chimiques et hydrobiologiques font l'objet du TABLEAU I, leur interprétation selon la technique des systèmes de saprobité fait l'objet du TABLEAU II.

Il est certain que les résultats présentés dans cette note doivent être interprètés en fonction de la dynamique de l'écosystème au moment des échantillonnages.

Passons rapidement en revue tout le cours du fleuve :

1. L'Yser à la frontière est dans un état relativement sain. L'index étendu indique une situation oligosaprobie. Il n'y a pas de développement excessif du phytoplancton.

Pourtant, la teneur en phosphates est déjà élevée. On trouve plus de $3 \mathrm{mg}$ de phosphore par litre (exprimé en ion phosphate (8), ce qui est élevé.

En hiver, le développement sur plaquettes montre une situation $\beta-\alpha$ mésosaprobie. II est vraisemblable que les nitrates sont le facteur limitant en été, comme c'est d'ailleurs le cas pour tout le reste de l'Yser. 
2. L'index étendu pour le Heidebeek indique un caractère isosaprobie. On perçoit cependant une poussée du développement planctonique, surtout des Chlorococcales, mais aussi une absence d'oxygène et une faible concentration en nitrates. On y rencontre des concentrations importantes en matières organiques et phosphates. La pollution bactérienne est nettement plus importante que dans l'Yser.

L'examen hydrobiologique montre en hiver une prèsence importante de Peritriches : Carchesium polyspinum et de Bacillariophycées : Navicula sp., Nitzschia sp. et Surirella ovata, insensibles aux fortes variations en oxygène. Le milieu est $\alpha$-mésosaprobie ou fort pollué.

3. Un deuxième affluent, le Harringebeek, fort pollué par une usine de préparation de pommes de terre, ainsi que par les égouts de Roesbrugge, a aussi une forte influence sur l'Yser. L'index étendu est métasaprobie. La population planctonique est fort réduite et le milieu est même nocif aux Escherichia coli et aux coliformes dont le nombre est supérieur en amont de l'usine.

Pendant l'hiver, la situation est anormale; on y trouve des quantités massives de Saccharomyces (fungi), en rapport vraisemblablement avec la présence de déchets de pommes de terre dans l'eau provenant d'une industrie. La biocénose indique un milieu hypersaprobie.

4. En aval de ces deux affluents, l'Yser devient nauséabond. En conséquence de la faible vitesse d'écoulement, les apports en matières organiques se dispersent difficilement, ce qui mène à des situations locales d'anaérobiose.

L'index étendu indique des circonstances isosaprobies. En effet, on observe une diminution en $\mathrm{O}_{2}$ et $\mathrm{NO}_{3}^{-}$et une augmentation en matières organiques et en éléments nutritifs ( $N$ total, en majorité $N$-organique, et $P$ ).

L'examen des biocénoses montre l'apparition de bactéries (Thiopedia rosea. Chromatium), et la prédominance des consommateurs (ciliates : surtout Carchesium polyspinum, Vorticella sp, Uronema marinum et autres ciliés). Le milieu est ici nettement $\alpha$-mésosaprobie.

\section{En aval de cette zone il y a récupération}

On observe un développement croissant du phytoplancton jusqu'à Dixmude après la confluence avec le canal de Poperinge, le Kemmelbeek et principalement le canal d'Ypres

Les Chloroccoccales dominent et les communautés indiquent des situations $\beta$ - $\alpha$ mésosaprobies.

6. Le canal d'Ypres ( $\mathrm{km}$ 17,5), principal affluent de l'Yser, reçoit les plus grandes charges d'eaux domestiques et de rejets d'élevages et de bio-industries de la région d'Ypres.

L'eutrophisation y est très prononcée et la densité planctonique exceptionnellement élevée (Chlorophycées et Bacillariophycées). Grâce à l'écoulement lent des eaux de ce canal et à l'autoépuration, l'influence sur l'Yser, à la confluence, n'est pas manifeste.

7. L'influence sur l'Yser des 3 derniers affluents se solde par une augmentation des teneurs en tous les éléments nutritifs et une tendance $\beta$-mésosaprobie de la communauté vivante (Chlorococcales), indiquant des processus d'oxydation (en parallèle, augmentation de la teneur en nitrates). Les conditions se trouvent ainsi réunies pour provoquer la prolifération du plancton, ce qui est bien observé. 
8. A la sortie de Dixmude, le Handzamevaart, très pollué par de nombreux rejets tout le long de son cours et, peu avant la confluence, par les eaux résiduaires de Dixmude rejetées sans épuration, a une influence néfaste sur l'Yser.

L'état d'anaérobiose de ses eaux provoque dans I'Yser l'empoisonnement de la masse énorme de phytoplancton par la formation des gaz nocifs. $\mathrm{H}_{2} \mathrm{~S}$ et $\mathrm{NH}_{3}$.

Des bactéries sulfureuses (Beggiatoa alba) et filamenteuses (Sphaerotilus natans), typiques d'une eau polysaprobie, dominent et donnent à l'eau une couleur vert-noire.

La pollution bactérienne augmente également très nettement dans la région de Dixmude.

La forte pollution observée ici dans I'Yser est due, moins à l'apport de matières organiques du Handzamevaart, qu'à la putréfaction du plancton qui résulte de cet apport.

9. En amont de Nieuport une eutrophisation avancée est de nouveau apparente.

\section{SOURCES DES ELEMENTS NUTRITIFS DANS LE BASSIN DE L'YSER}

L'examen des sources d'éléments nutritifs montre que l'agriculture et surtout l'élevage sont en cause.

En effet, le bassin de l'Yser se trouve dans une région à faible densité de population ( $180 \mathrm{hab} . / \mathrm{km}^{2}$ ), la population totale du bassin étant de 125000 habitants, répartie en agglomérations rurales de moins de 2500 hab. Quelques centres comme Poperinge et Ypres ont plus de 10000 habitants. Quoique les eaux usées ne soient pas épurées, sauf dans la région de Nieuport (8) et contiennent des matières nutritives dont le phosphore des détergents, cette source d'éléments nutritifs ne peut être essentielle en raison du petit nombre d'habitants (Fig. 2).

II n'y a pas d'industries chimiques rejetant dans l'Yser des eaux résiduaires toxiques. La plupart des industries de la région sont des industries alimentaires (laiteries, brasseries, conserveries, exploitations de déchets d'abattoirs, etc.) qui rejettent plus d'éléments nutritifs dans leurs eaux résiduaires que les autres types d'industries (7).

Ce secteur contribue vraisemblablement pour une part importante à l'eutrophisation (Fig. 3).

La région de l'Yser est donc essentiellement agricole. La superficie cultivée représente $58,5 \%$ de la partie agricole du Bassin. Le cheptel est très important vis-à-vis des dimensions de la région. On dénombre 140000 bovidés et 550000 porcins (9). Deux types de pollution peuvent être distingués : la pollution indirecte, provenant du lessivage des fertilisants répandus sur les terres cultivées, du ruissellement et de l'érosion; les rejets directs (élevage) dans le cours d'eau, qui provoquent les pollutions les plus graves (10) (Fig. 4). 


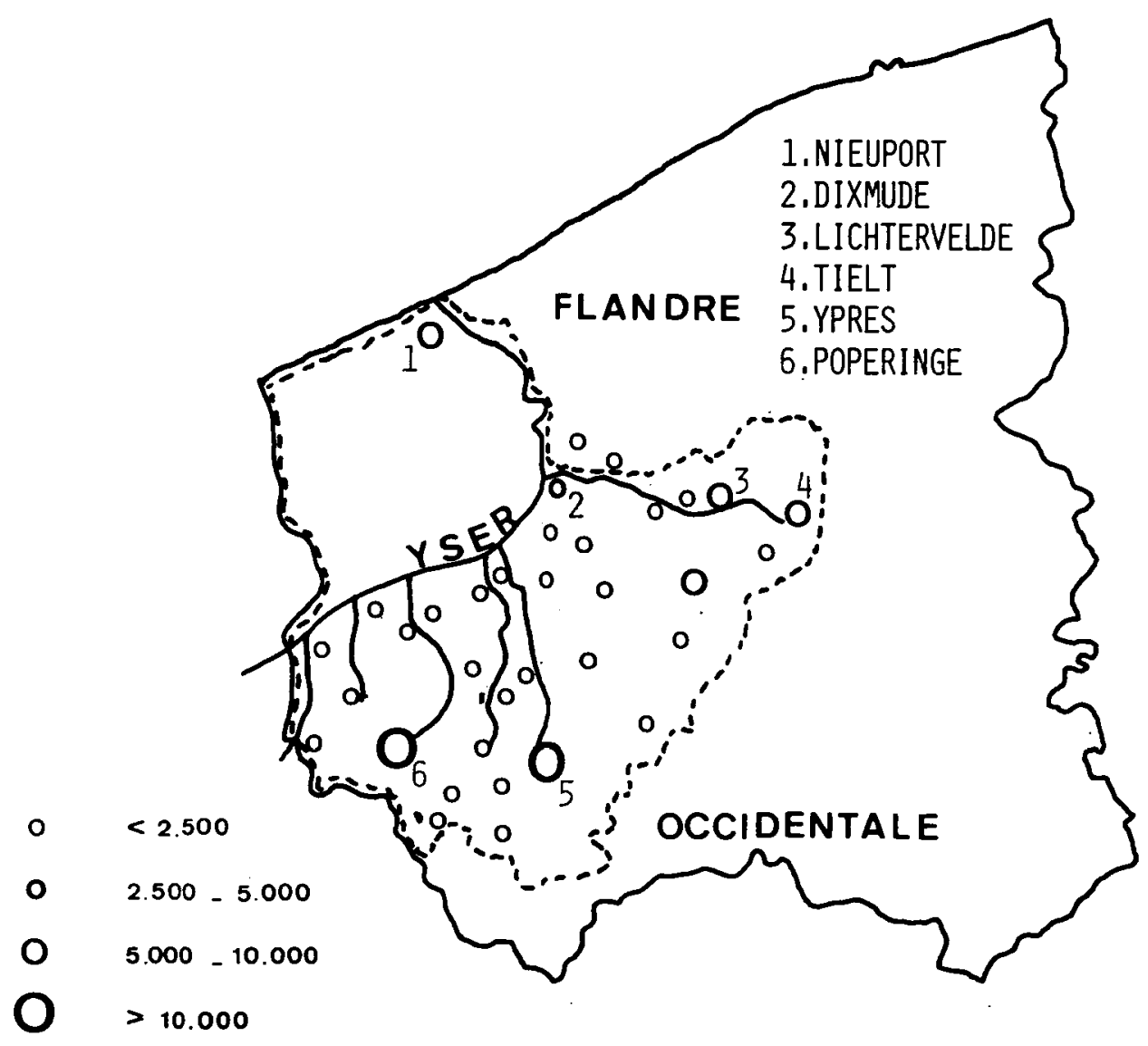

Fig. 2 - Population du Bassin de l'Yser. 


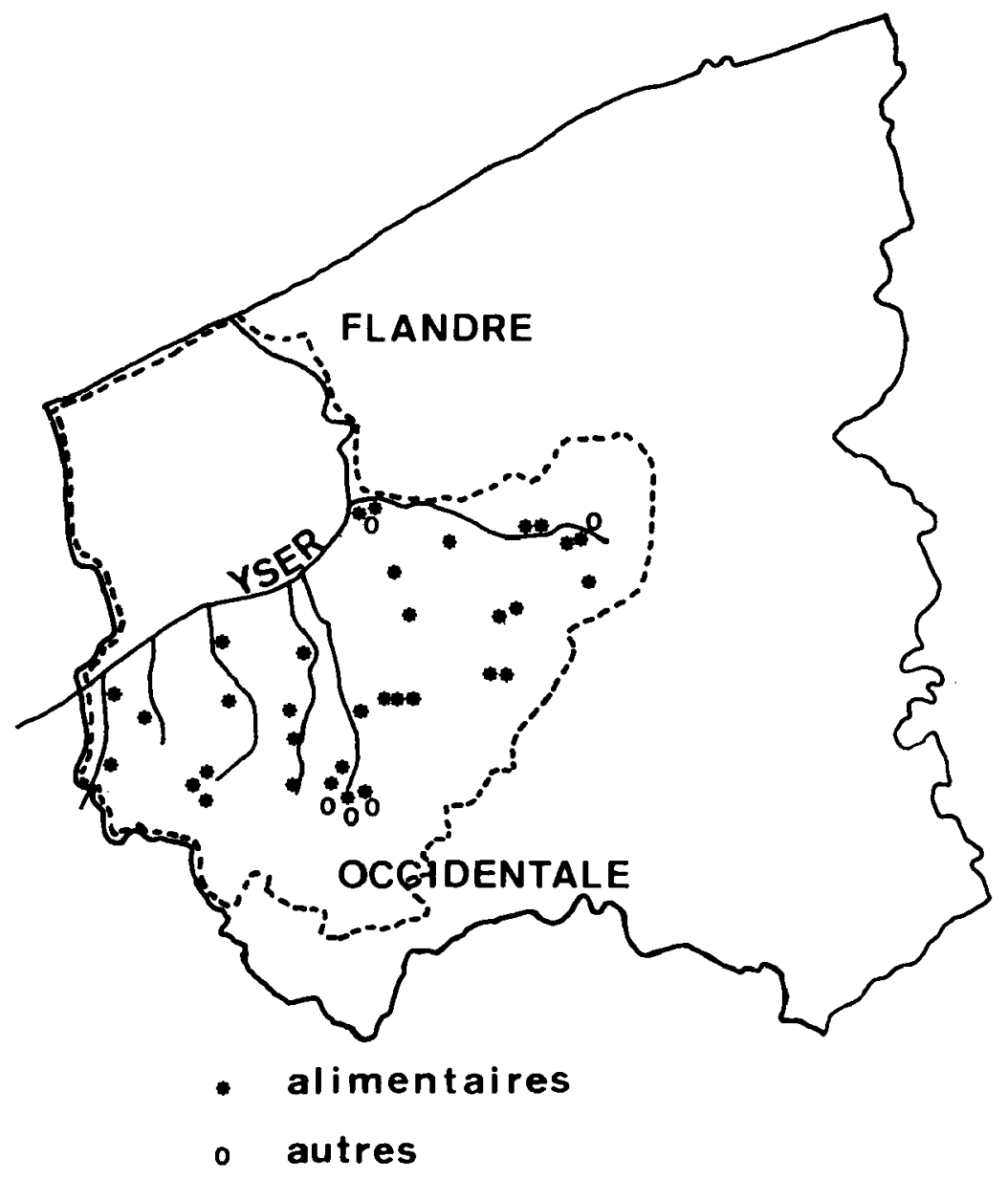

Fig. 3 - Industries du Bassin de l'Yser. 


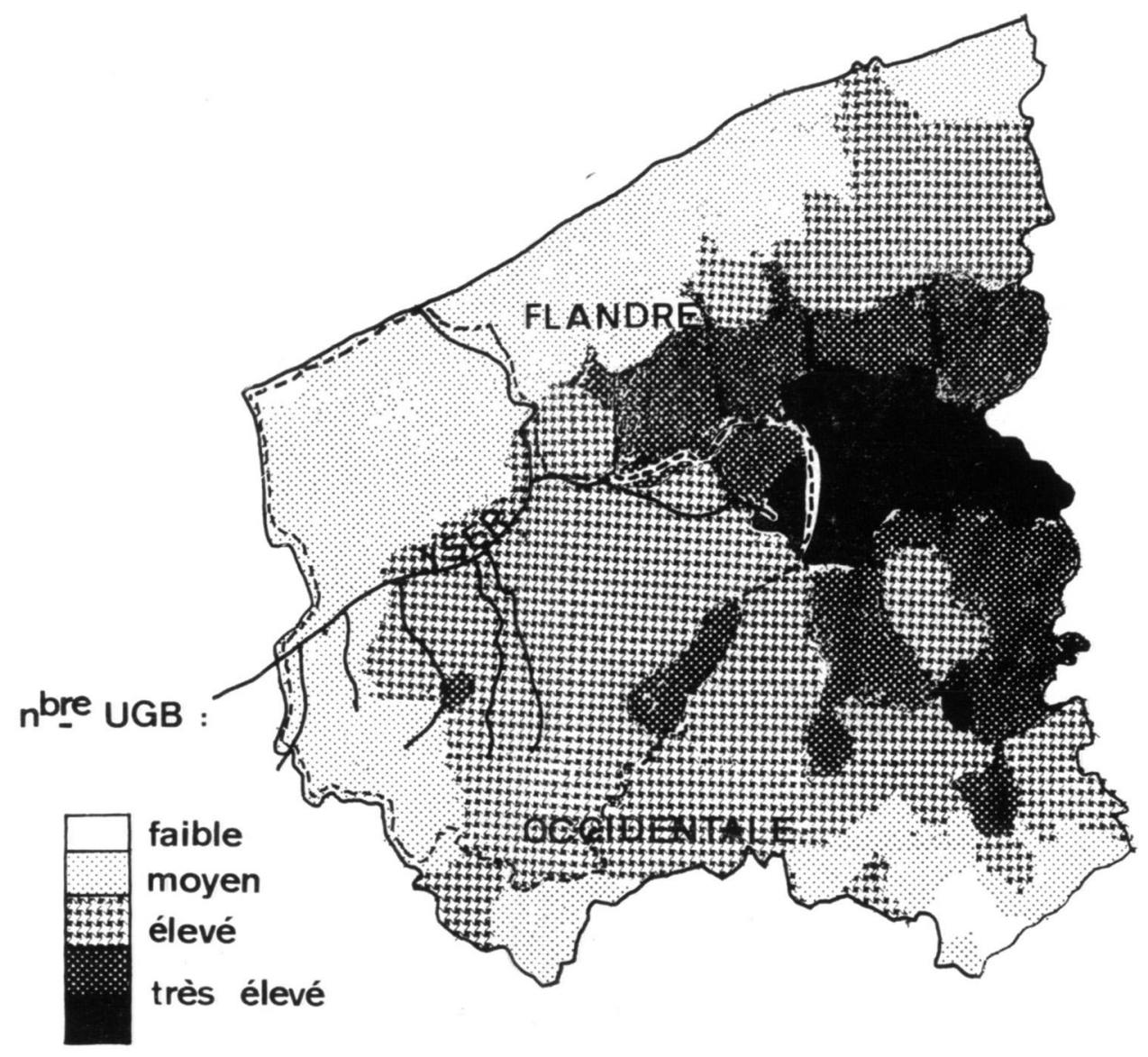

Fig. 4 - Elevages du Bassin de l'Yser. 


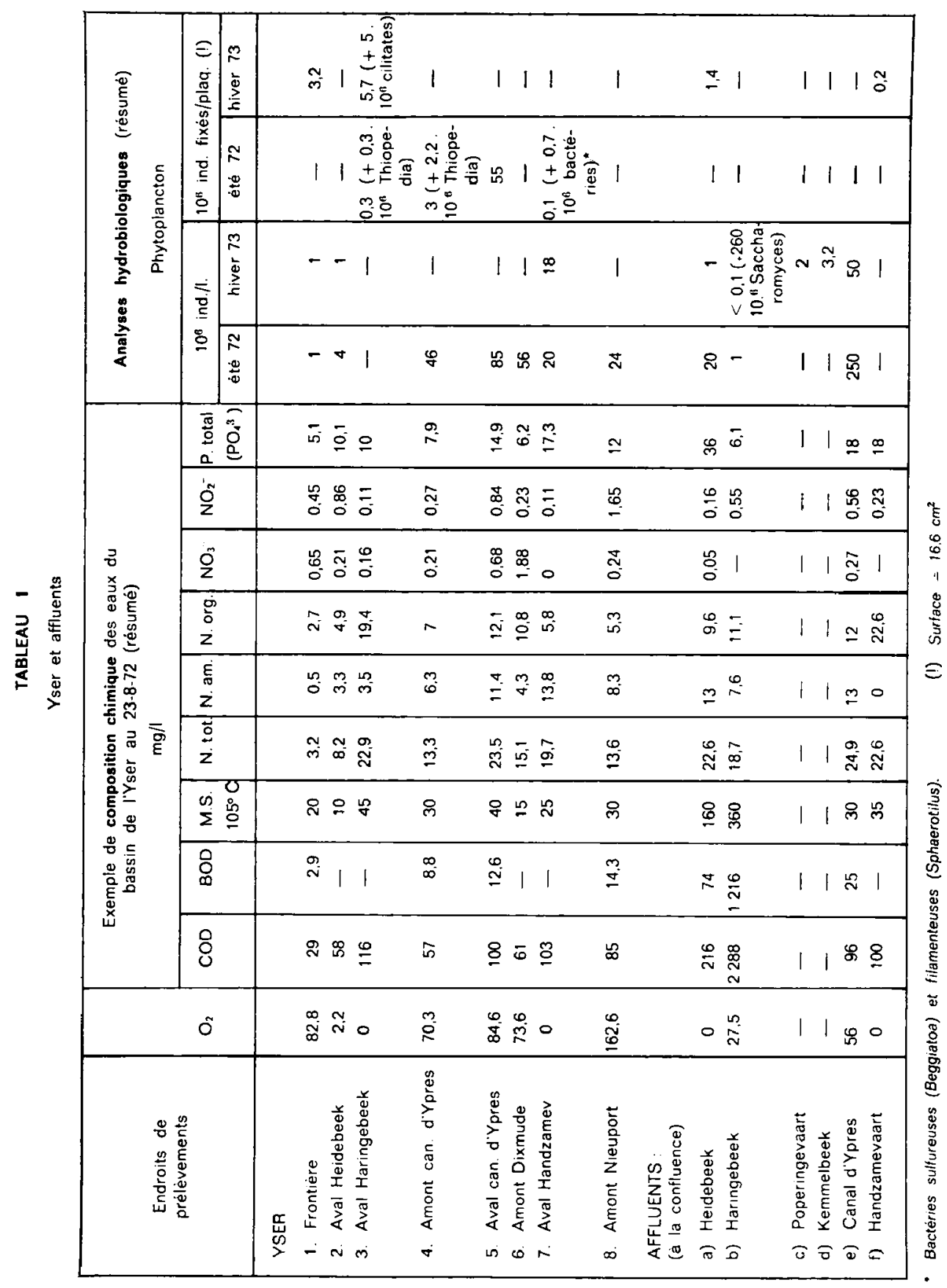




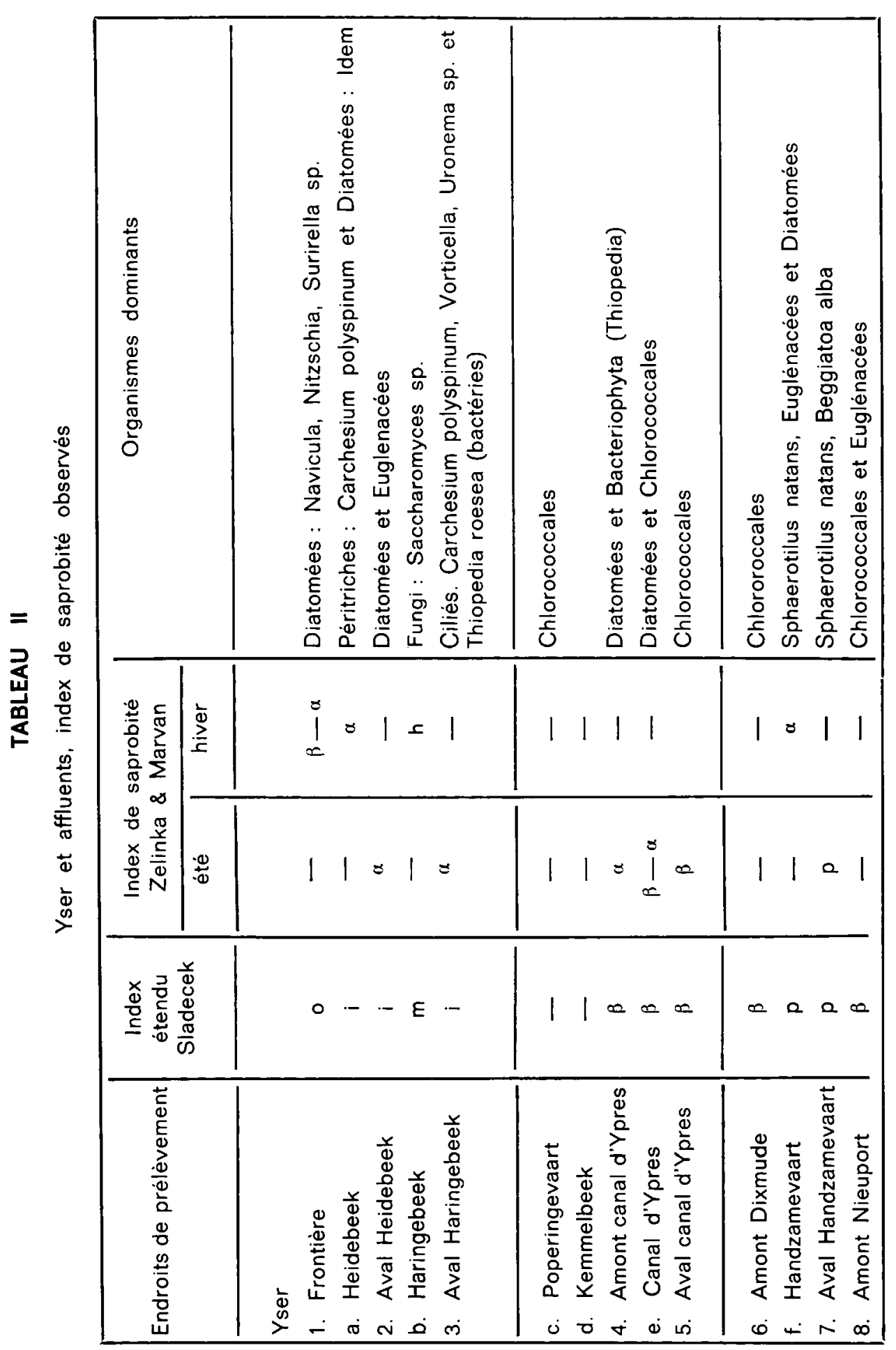




\section{CONCLUSIONS}

L'examen hydrobiologique fournit une image claire de l'eutrophisation de tout l'Yser, conséquence de la forte concentration en éléments nutritifs. En fait, le stade eutrophe est dépassé et les caractéristiques typiques de pollution autogène apparaissent sous forme notamment de fleurs d'eau, d'anaérobiose, de gaz de putréfaction, etc., expliquant la détérioration du bassin de I'Yser.

Le fait qu'il s'agisse d'un phénomène biologique à propriétés cycliques, liées à la température, à la luminosité, au débit, à la salinité et à d'autres facteurs écologiques, explique sans aucun doute les mortalités massives de poissons signalèes régulièrement.

\section{REMERCIEMENTS}

Pour m'avoir aidé à réaliser cette note, je remercie :

M. J. BOUQUIAUX, Chef du Département de I'Environnement. miologie.

Mme GOBERT-DE SCHUTTER, laborantine à l'Institut d'Hygiène et d'EpidśJe remercie également Mme MEEUS de l'Institut de Recherches chimiques.

\section{BIBLIOGRAPHIE}

(1) De ljzer ICWB (IHE-IRC) (Nov. 1973) 1 Vol. 105 p.

(2) Service d'études hydrologiques. Ministère Travaux publics.

(3) Environmental monitoring series : Biological field and laboratory methods for measuring the quality of surface water and effluents. Ohio, EPA670/4-73.001 (July 1973).

(4) ZELINKA, M. \& MARVAN, P. Zur Präzisierung der biologischen Klassifikation der Reinheit Fliessender Gewässer.

Arch. Hydrobiol. (1961) 57,389

(5) SLADECEK, V. : Technische Hydrobiologie II. Tschechoslowakische Beiträge zum Saprobiensystem. Scientific papers from Institute of chemical Technology of Water (1964) 8 (1), 529.556.

(6) SLADECEK, V. : System of Water Quality from the Biological Point of View. Arch. für Hydrobiol. (1973) 7, I-IV, 1-218.

(7) OCDE : Recherches sur l'aménagement de l'eau (1968).

(8) BEERNAERT, S. (1973). De waterverontreiniging in het lizerbekken. Facetten van West-Vlaanderen, Westvlaams Economisch Studiebureau, Brugge.

(9) Institut national de Statistiques. Recensement agriculture et horticulture du 15 mai 1972. Ministère des Affaires économiques.

(10) MEEUS-VERDINNE, K. : Eutrophisation dans le Bassin de l'Yser. Semaine d'étude agriculture et environnement.

Centre Recherches agronomiques Gembloux (1974) 153-167. 